\title{
In vitro preservation of traumatic human skin autografts in a massive degloving injury
}

\author{
JAMES E. HOOGEBOOM, DO \\ JOHN W. POLLEY, MD \\ JOSEPH P. HUNSTAD, MD \\ DONALD J. SCHOLTEN, MD \\ RANDALL TELMAN, MD
}

\begin{abstract}
The in vitro preservation of human skin autografts is a valuable technique in the management of severe degloving injuries. The authors describe the storage of traumatically induced human skin autografts involving nearly one third of the total body surface of a patient. These autografts were preserved in tissue-culture medium for 19 days and then transplanted onto the patient's prepared wounds, successfully acting as biologic dressings and permanent grafts. To the authors' knowledge, this is the largest clinical application of skin-storage techniques for the preservation of traumatic human skin autografts that is known to have been reported.
\end{abstract}

The preservation and short-term storage of human skin autografts is advantageous in many clinical situations. The current methods for skin storage above $0^{\circ} \mathrm{C}$ have been developed largely through controlled clinical studies ${ }^{1-5}$ and the use of experimental animal models. ${ }^{6-11}$ In this report, we document the successful processing and in vitro preservation of traumatically produced human skin autografts that involved nearly $30 \%$ of a patient's total body surface. These autografts were subsequently used as biologic dressings and permanent grafts.

\section{Report of case}

A 24-year-old man suffered severe pelvic and lowerextremity injuries when his motorcycle slid underneath a moving gravel truck. Injuries included open pelvic frac- tures and dislocation, anorectal avulsion, urethral disruption, multiple upper-extremity lacerations and abrasions, and a massive degloving injury of both lower extremities (Fig 1). On the right leg, the skin was avulsed in a circumferential fashion, beginning above the iliac crest, including the entire hip, thigh, and leg, and ending at the ankle. A similar injury involved the left leg, with more skin spared on the left thigh. The degloved skin, approximating $30 \%$ of the patient's total body surface, remained attached through small skin bridges at the distal aspect of the abdomen and at both ankles (Fig 2). All wounds were heavily soiled, and neurovascular structures were exposed in both popliteal fossae and the right femoral canal.

Initial management was started in the trauma room. Rapid fluid resuscitation with crystalloid and packed red blood cells was initiated. Large-bore venous catheters were placed, and an arterial line was inserted for pressure monitoring. Chest and pelvic $\mathrm{x}$-ray films were obtained. Tetanus prophylaxis and a broad-spectrum antibiotic regimen were started. Blood was drawn for laboratory tests and cross-matched for multiple units of blood products.

The patient was then rapidly moved to the operating room. All wounds were quickly inspected and hemostatis obtained. Fluid therapy was continued to maintain adequate perfusion of viable tissue. Because of familial diabetes insipidus, urine production was not an adequate indicator of volume status in this patient. A multidisciplinary approach was used to expedite surgical care. The trauma team performed a laparotomy. The urology service then performed debridement and reanastomosis of the urethral disruption. Later, a suprapubic cystostomy was placed to protect the urethral anastomosis and to assure bladder decompression. The orthopedic service reduced and plated the diastasis of the pubic symphysis to improve pelvic stabilization and control hemorrhage from the pelvic fractures. 

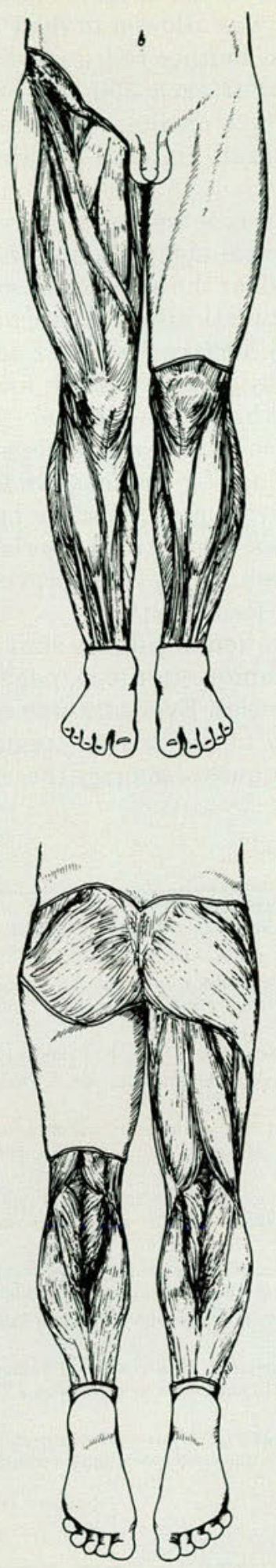

Figure 1. Anterior (top) and posterior ( bottom) views of degloving injury; the shaded area denotes full-thickness skin loss.

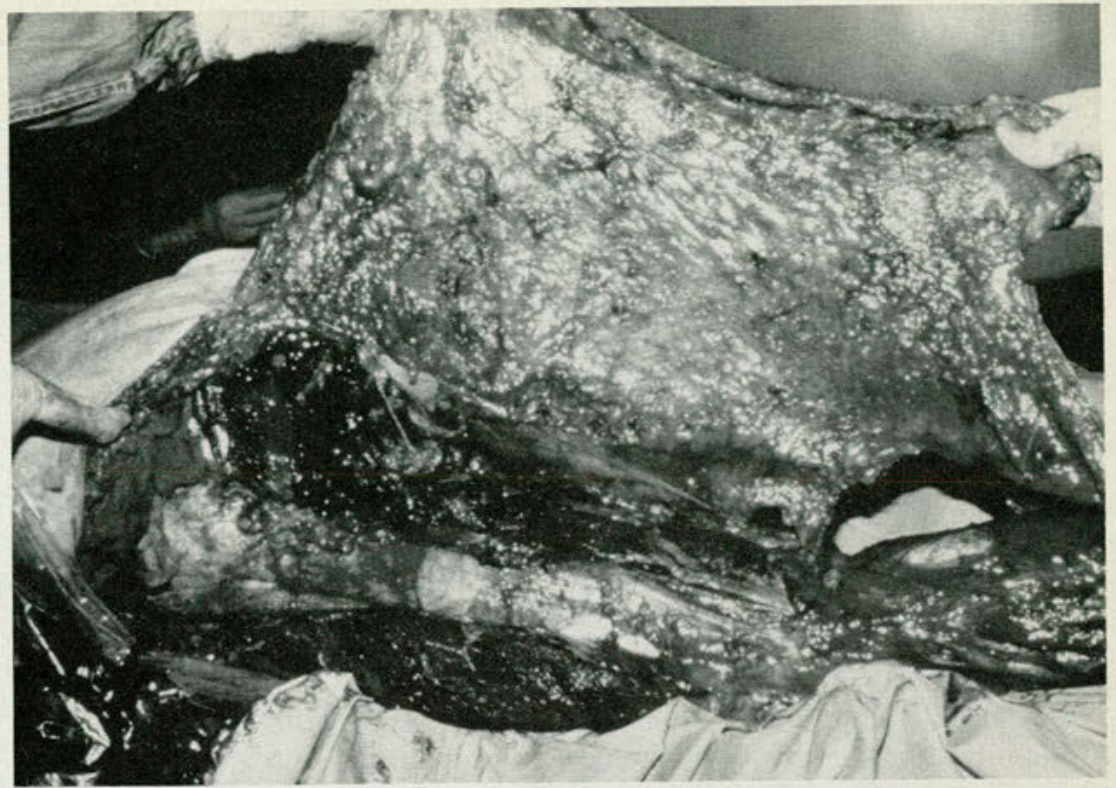

Figure 2. Massive sheet of degloved skin remained attached at the abdomen and ankle.

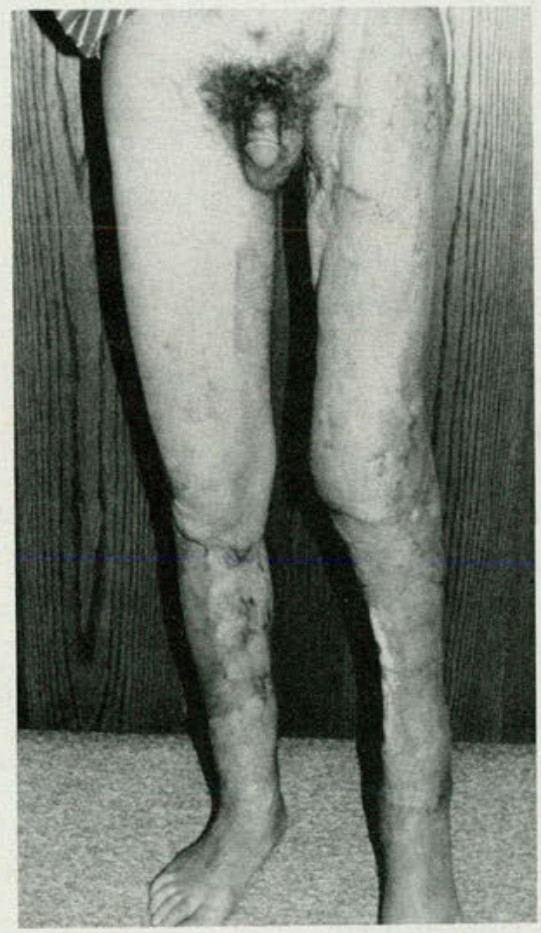

Figure 3. Patient at 8 months postinjury.
A sigmoid end colostomy and mucous fistula were created because the anorectal avulsion had completely disrupted the sphincter mechanism. In addition, with the massive soft-tissue injury to both legs as well as to the perineum and buttocks, diversion of the fecal stream to avoid gross contamination was considered essential.

The plastic surgery team worked simultaneously with the abdominal surgery team. Debridement and lavage of the lower-extremity wounds were performed, and the skin flaps were washed with a salinechlorhexidine gluconate (Hibiclens) solution. Intravenous fluorescene was used to determine the limit of viable skin. Using an electric dermatome, we harvested 30 split-thickness skin grafts $(0.033 \mathrm{~cm})$ totaling $3800 \mathrm{~cm}^{2}$ from the nonstaining skin. The grafts were placed on gauze sponges moistened with McCoy's tissue-culture medium with a low concentration of neomycin $(25 \mathrm{\gamma g} / \mathrm{mL})$ and penicillin $(50 \mathrm{U} / \mathrm{mL})$, and buffered to a $\mathrm{pH}$ of 7.0. The grafts were stored at $4^{\circ} \mathrm{C}$ and periodically rebathed in fresh McCoy's medium, based on the $\mathrm{pH}$ color indicator in the medium. 
Postoperatively, the patient experienced frequent episodes of bacteremia, with blood and urine cultures positive for Pseudomonas aeruginosa. Initial wound biopsies revealed bacterial counts of 100,000 organisms per gram of tissue. Daily dressings with silver sulfadiazine were placed, and on four separate occasions, the patient was returned to the operating room for aggressive wound debridement. On hospital day 19 , the stored skin grafts were rinsed in sterile saline solution and transplanted onto the prepared lower-extremity wounds. Approximately $75 \%$ of the original wounds were covered with the preserved skin; the remaining wounds were covered with split-thickness skin grafts harvested from the patient's back.

Following wound coverage, the patient's clinical condition steadily improved, with almost $50 \%$ of the preserved grafts retaining viability. The remaining areas were successfully regrafted at a later date.

On hospital day 101, the patient was discharged to a rehabilitative institution. After 3 months of rehabilitative therapy, the patient is again leading an ambulatory life-style. Figure 3 shows the patient 8 months postinjury.

\section{Discussion}

The short-term preservation of human skin dates back to 1902 , when Wentscher ${ }^{1}$ reported the autografting of skin refrigerated for periods of up to 14 days. In 1944, Webster ${ }^{2}$ reported the successful transplantation of human skin that had been wrapped in saline-soaked gauze and refrigerated at $4^{\circ} \mathrm{C}$ for up to 21 days. Skoog ${ }^{6}$ and Georgiade and colleagues, ${ }^{7}$ however, found that after 20 days, cellular respiration had ceased in refrigerated skin stored in only saline solution. Marrangoni ${ }^{3}$ recommended a storage solution of $10 \%$ serum for refrigerated skin autografts.

It was not until 1983 that the preservation of human skin autografts in tissue culture medium was first reported. ${ }^{8,9}$ Using the nude mouse model, Cram and colleagues ${ }^{8,9}$ reported the predictably reliable survival of human skin autografts refrigerated in tissue culture medium for up to 22 days. They demonstrated the superiority of a medium containing the basic requirements for cellular metabolism over saline as a storage solution. They also emphasized the importance of adding a low concentration of antibiotics, maintaining a medium $\mathrm{pH}$, and periodically replenishing the grafts with fresh medium. In a 1985 update, Cram and colleagues ${ }^{10}$ again showed the superiority of tissue culture medium over saline solution for the refrigerated storage of viable human skin.

More recently, Rosenquist and colleagues ${ }^{11}$ have demonstrated a modification of the tissue culture skin-storage technique that has improved graft survival. The stored skin was allowed to float freely in the medium and was neither rolled nor backed with nylon or gauze. For each $300 \mathrm{~cm}^{2}$ of skin stored, $100 \mathrm{~mL}$ of culture medium was used. At 30 days storage time, graft viability remained at $50 \%$.

To our knowledge, the case we report represents the most extensive clinical application of the current methods developed for the short-term storage and preservation of traumatically produced human skin autografts. Nearly $4000 \mathrm{~cm}^{2}$ of degloved skin was preserved in McCoy's tissue culture medium at $4^{\circ} \mathrm{C}$ and transplanted back to the patient 19 days following injury. These autografts successfully acted as biologic dressings for the massive lowerextremity wounds, providing an effective barrier to vapor and exudate loss, preventing bacterial contamination, aiding in pain control, and preventing exuberant granulation formation.

The in vitro preservation of human skin autografts is a valuable technique in the management of severe degloving injuries. Every trauma center should have the ability to use current tissue culture preservation techniques to manage these massive injuries.

1. Wentscher J: Ein weiterer beitrag zur uberlebensfahikeit der men schlichen epidermiszellen. Dietsche Zeitsher P Chir 1903;70:21.

2. Webster JP: Refrigerated skin grafts. Ann Surg 1944;120:431

3. Marrangoni AG: An experimental study on refrigerated skin grafts stored in 10 percent homologous serum. Plast Reconstr Surg 1950;6:425434 .

4. Perry VP: A review of skin preservation. Cryobiology 1966;3:109-130. 5. Lawrence JC: Storage and skin metabolism. Br J Plast Surg 1972;25:440-453.

6. Skoog T: An experimental and clinical investigation of the effect of low temperature on the viability of excised skin. Plast Reconstr Surg 1953; $14: 403$.

7. Georgiade N, Peschel E, Georgiade R, et al: A clinical and experimental investigation of the preservation of skin. Plast Reconstr Surg 1956;17:267-275.

8. Cram AE, Domayer MA, Shelby J: Human skin storage techniques: A study utilizing a nude mouse recipient. J Trauma 1983;23:924-926.

9. Cram AE, Domayer MA, Short-term preservation of human autografts. J Trauma 1983;23:872-873.

10. Cram AE, Domayer MT, Scupham R: Preservation of human skin: A study of two media using the athymic (nude) mouse model. $J$ Trauma $1985 ; 25: 128-130$

11. Rosenquist MD, Cram AE, Kealey GP: Short-term skin preservation at $4^{\circ} \mathrm{C}$ : Skin storage configuration and tissue-to-volume medium ratio. J Burn Care Res 1988;9:52-54.

From the department of surgery, Butterworth Hospital, Grand Rapids, Mich.

Reprint requests to Dr Hoogeboom, 100 W Third Ave, Columbus, OH 43201. 